\title{
Consensus Problem in V2V-based Vehicular Ad Hoc Networks
}

\author{
Chien-Fu Cheng ${ }^{+}$ \\ Department of Computer Science and Information Engineering, Tamkang University, New Taipei, Taiwan
}

\begin{abstract}
Intelligent vehicles use Vehicular Ad Hoc Networks (VANETs) to communicate with other intelligent vehicles. However, messages are transmitted wirelessly in VANET and therefore vulnerable to malicious attacks. In the event of a malicious attack, the On-Board Unit (OBU) in intelligent vehicles will not function properly, and accidents are likely to occur. Therefore, building a highly reliable and fault-tolerant VANET is of high important. In this paper, based on the characteristics of Vehicle-to-Vehicle (V2V) based VANETs, we design a fault-tolerant consensus protocol to improve fault tolerance of V2V-based VANETs.
\end{abstract}

Keywords: vehicular ad hoc networks, vehicle-to-vehicle, fault-tolerant, consensus problem.

\section{Introduction}

The Intelligent Transportation Systems (ITS) equipped in intelligent vehicles is called On-Board Unit (OBU). An OBU consists of a CPU, a set of transceiver, and a GPS. Via vehicular ad hoc networks (VANETs), vehicles installed with an OBU can communicate with one another. In addition to OBUs, RoadSide Unit (RSU) is another important component of VANETs. RSUs can offer Internet access to OBUs by connecting to the back-end network [22]. Common applications of ITS include traffic control and forecast [10][20], navigation system [4][21], electronic toll collection [3][14], multimedia streaming [16][18], and etc.

In VANETs, communications are based on two distinct models, including Vehicle-to-Vehicle (V2V) [7][12] and Vehicle-to-Roadside (V2R) [1][5]. V2V refers to communications between OBUs. This model is suited for use by vehicles that are within the communication range of each other. It is a non-infrastructurebased communication model. V2R refers to communications between OBUs and RSUs. Given two vehicles that are not within the communication range of each other, the sender OBU can send the message to a nearby RSU, which will relay the message to an RSU nearby the receiver OBU, and this RSU will further deliver the message to the receiver OBU. In other words, V2R is infrastructure-based.

In VANETs, messages are transmitted wirelessly, so the network may be vulnerable to malicious attacks. When being maliciously attacked, an OBU may malfunction and even conspire with other OBUs under attack to send falsified messages to interfere with the normal operation of the entire network. The network will then become paralyzed and produce wrong computing results [19][22], which may lead to occurrence of car accidents. Therefore, building a fault-tolerant and highly reliable VANET is important. Generally, faults in VANETs can be classified into cash faults [11][15] and Byzantine faults [13][17]. A crash fault means that the device will stop operating. However, on the occasion of a Byzantine fault, the behavior of a faulty device is unpredictable and arbitrary. One of the most well-known problems in fault-tolerant distributed system is the consensus problem [2]. This problem is defined as follows: (1) the network consists of $n$ processors $(n \geqq 4)$, with no more than $t(t=\lfloor(n-1) / 3\rfloor)$ faulty processors; (2) all processors do not have knowledge of which ones of them are faulty; (3) processors communicate via a reliable fully connected network; (4) the receiver is able to identify the sender; (5) of these $n$ processors, each processor has an initial value and exchanges messages to obtain a consensus value. The main challenge in this problem is that

+ Corresponding author. Tel.: +886-2-2621-5656 ext. 2668

E-mail address: cfcheng@mail.tku.edu.tw 
Byzantine fault may occur. For Byzantine faulty processors, conspiracy is the worst type of fault. Conspiracy occurs when a hacker or a virus causes processors to be eavesdropped or controlled and then work with other Byzantine faulty processors to interfere with the normal operation of correct processors. The objective of the research of the consensus problem is to design a protocol that assists processors in the system to achieve a consensus through a minimum number of rounds of message exchange while tolerating the maximum number of faulty components in the system. In this paper, we will discuss how to address the consensus problem in V2V-based VANETs.

\section{System Model}

In previous study, Fischer et al. [9] have proven that the consensus problem cannot be solved in a strictly asynchronous system. In the strictly asynchronous system, there is no assumption on communication delays and relative speed of processors, and no access to real-time clocks. In view of this, the consensus problem is discussed in an asynchronous VANET with the partially synchronous assumption [6]. The VANET under consideration consists of a set of vehicles $O$. Each vehicle has an initial value, and the domain range $D$ $=\{0,1\}$. Each vehicle and in the network can be identified uniquely, and a correct vehicle does not know the faulty status of other vehicles in the network. We assume that the failure type of faulty vehicles is Byzantine fault [13][17]. This implies that the behavior of the Byzantine faulty vehicle is unpredictable. All the messages are encrypted during transmission, so it is impossible that relay devices falsify a message from its sender to its receivers. But, the Byzantine sender may send inconsistent messages to different vehicles.

\section{The Concept and Approach}

In V2V-based VANETs, no RSU is involved in the data transmission process, and vehicles need to rely on multi-hop transmission to communicate with one another. Because vehicles move at a high speed, the topology also varies quickly. If vehicles have to temporarily exit the network for certain reasons during execution of the consensus protocol, these away vehicles will not be able to collect sufficient messages and thus fail to reach a common consensus value after returning to the network. Hence, we need to design a consensus protocol for $\mathrm{V} 2 \mathrm{~V}$-based VANETs that allows vehicles that have temporarily exited from the network during execution of the algorithm to reach a common consensus value after returning to the network. In this section, we propose a consensus protocol to solve the consensus problem in the V2V-based VANET. The proposed protocol consists of two phases, including message exchanging phase and consensus making phase.

\subsection{Message Exchanging Phase}

The message exchanging phase is used to collect the messages from other vehicles. In this subsection, we will explain the data structure, handling of lost messages and the number of rounds required of the proposed consensus protocol.

\subsubsection{Data Structure}

During the execution of consensus protocol, each vehicle will store the received messages into a matrix data structure. The matrix is called CP-matrix. In Fig. 1, we introduce CP-matrix from the viewpoint of vehicles $o_{0}$. Assume there are 7 vehicles, respectively denoted by $o_{0} \sim o_{6}$, in the network. When vehicle $o_{0}$ receives an initial value of another vehicle, it will store the initial value in its CP-matrix $\mathcal{M}^{1}\left(o_{0}\right)$. As shown in the upper left part of Fig. 1, because there are 7 vehicles, there will be 7 elements in $\mathcal{M}^{1}\left(o_{0}\right)$, where value $(i)$ in $\mathcal{M}^{1}\left(o_{0}\right)$ denotes that the value comes from $o_{i}$ and is stored in $\mathcal{M}^{1}$ of vehicle $o_{0}$ (value $(i)$ can also be expressed as $\left.\mathcal{M}^{1}\left(o_{0}\right)[i]\right)$. When vehicle $o_{0}$ receives the value $\mathcal{M}^{1}\left(o_{i}\right)$ of vehicle $o_{i}$, it will store the value $\mathcal{M}^{1}\left(o_{i}\right)$ in its CP-matrix $\mathcal{M}^{2}\left(o_{0}\right)$. As shown in the lower left part of Fig. 1, because there are 7 elements in its $\mathcal{M}^{1}\left(o_{i}\right)$, there will be 49 elements in $\mathcal{M}^{2}\left(o_{0}\right)$, where value $(j, k)$ in $\mathcal{M}^{2}\left(o_{0}\right)$ denotes that the value is passed from $o_{j}$ and $o_{k}$ and is stored in $\mathcal{M}^{2}$ of vehicle $o_{0}$ (value $(j, k)$ can also be expressed as $\mathcal{M}^{2}\left(o_{0}\right)[j][k]$ ). When vehicle $o_{0}$ receives the value $\mathcal{M}^{2}\left(o_{i}\right)$ of vehicle $o_{i}$, it will store the value $\mathcal{M}^{2}\left(o_{i}\right)$ in its CP-matrix $\mathcal{M}^{3}\left(o_{0}\right)$. Given 7 vehicles, vehicle $o_{0}$ will create 7 matrices including $\mathcal{M}^{3}\left(o_{0}\right)[0], \mathcal{M}^{3}\left(o_{0}\right)[1], \mathcal{M}^{3}\left(o_{0}\right)[2], \mathcal{M}^{3}\left(o_{0}\right)[3], \mathcal{M}^{3}\left(o_{0}\right)[4], \mathcal{M}^{3}\left(o_{0}\right)[5], \mathcal{M}^{3}\left(o_{0}\right)[6]$. The matrix $\mathcal{M}^{3}\left(o_{0}\right)[0]$ is shown in the right of the Fig. 1 , where value $(x, y)$ in $\mathcal{M}^{3}\left(o_{0}\right)$ [0] implies that the value is passed from $o_{0}$ to $o_{x}$ and then further passed to $o_{y}$ and is stored in $\mathcal{M}^{3}$ of vehicle $o_{0}$ (the value can also be 
expressed as $\left.\mathcal{M}^{3}\left(o_{0}\right)[0][x][y]\right)$. That is, after the first round of message exchange, each $o_{i}$ will create a matrix of its own $\mathcal{M}^{1}\left(o_{i}\right)$. After the second round of message exchange, each $o_{i}$ will create a matrix of its own $\mathcal{M}^{2}\left(o_{i}\right)$. This operation continues until the number of rounds of message exchange equals the number of rounds required.

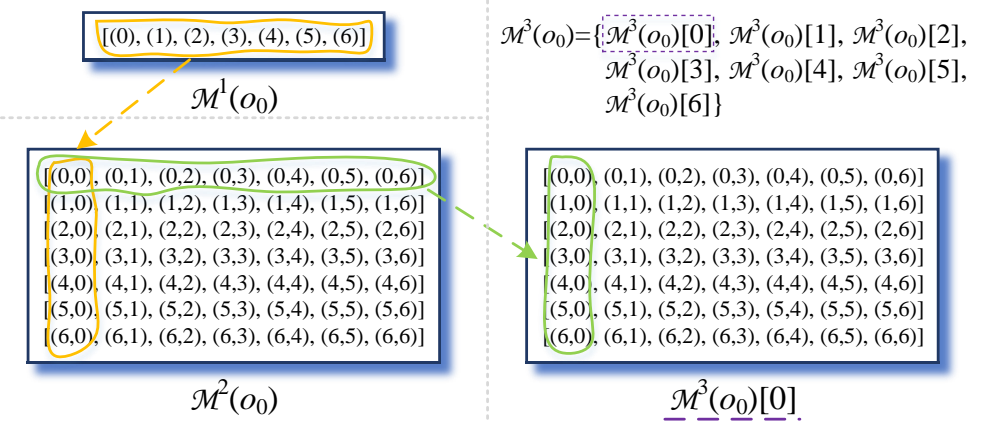

Fig. 1: Examples of CP-matrix.

\subsubsection{The Lost Messages from Away Vehicle}

In VANET, each vehicle can move freely. Hence, messages from away vehicles cannot be successfully received. The lost messages from away vehicles will be marked by $\zeta^{0}$. In the following rounds of message exchange, we need to inform other vehicles of vehicles with no value. Our method is as follows: if a vehicle receives $\zeta^{j}, \zeta^{j+1}$ instead of $\zeta^{j}$ will be stored and used to represent the missing value in the previous round. The purpose of the +1 design is to show how many rounds of message exchange have passed.

\subsubsection{The Number of Rounds Required of the Proposed Consensus Protocol}

We use the term round to compute the amount of message exchange. A round of message exchange comprises three steps as follows: (1) send messages to any set of receivers; (2) receive messages from other processors in this round; and (3) do local processing [8]. Moreover, Fischer et al. [8] point out that given a network consisting of some Byzantine faulty processors, if all the correct processors are unable to know which processor is faulty, and the number of Byzantine faulty processors is smaller than or equal to $t(t=L(n$ $1) / 3\rfloor$, where $n$ is the number of processors in the network), these correct processors can reach a consensus value after $t+1$ rounds of message exchange. Our assumption for the failure type of fallible processors (i.e. vehicles) and the definition of round are the same as those used in Fischer et al. [8]. So the maximum number of rounds required of the proposed consensus protocol is $\left\lfloor\left(n_{O^{-}}-1\right) / 3\right\rfloor+1$, where $n_{O}$ is the number of vehicles in the VANET.

\subsection{Consensus Making Phase}

The consensus making phase is used to compute the consensus value. In this subsection, we will explain how to calculate the consensus value for correct vehicles which have never exited the VANET (i.e. nonaway vehicles) and how return vehicles can obtain a consistent consensus value from other vehicles after returning the VANET.

\subsubsection{Calculating the Consensus Value for Non-away Vehicles}

After $\left\lfloor\left(n_{O^{-}}-1\right) / 3\right\rfloor+1$ rounds of message exchange, each correct vehicle $o_{i}$ that did not move away from the VANET during the message exchanging phase will create a matrix of its own CP-matrix $\mathcal{M}^{\left\lfloor\left(n_{o}-1\right) / 3\right\rfloor+1}\left(o_{i}\right)$. In the message exchange process, some values will be repeatedly forwarded by the same vehicle. To avoid repetitive interference, we delete values that are passed to the same vehicles. For example, $\mathcal{M}^{3}\left(O_{i}\right)[3][2][2]$ (i.e. element [3][2][2] in $\mathcal{M}^{3}\left(O_{i}\right)$ ) denotes a value that is passed from $o_{3}$ to $o_{2}$ and then further passed to $o_{2}$ again. We delete the value of $\mathcal{M}^{3}\left(O_{i}\right)[3][2][2]$. Later, we convert $\mathcal{M}^{\left\lfloor\left(n_{O}-1\right) / 3\right\rfloor+1}$ into $\mathcal{M}^{\left\lfloor\left(n_{O}-1\right) / 3\right\rfloor}$ using a majority function, and then convert $\mathcal{M}^{\left\lfloor\left(n_{O}-1\right) / 3\right\rfloor}$ into $\mathcal{M}^{\left\lfloor\left(n_{O}-1\right) / 3\right\rfloor-1}$. This conversion process continues until $\mathcal{M}^{0}$, which is the consensus value. It should be noted that the impact of lost messages should be considered in the design of the majority function. The proposed majority function is called vote matrix $_{\text {. The }}$ vote $_{\text {matrix }}$ function only counts the non-value $\zeta^{0}$ for all elements in CP-matrix. There are three conditions in the vote $e_{\text {matrix }}$ function. Condition (1): if the majority value is $\zeta^{j}$ then output the value $\zeta^{j-1}$, where $1 \leq j \leq\left\llcorner\left(n_{O^{-}}-1\right) / 3\right\rfloor$. 
Condition (2): if the majority value is non $\zeta^{j}$ value then output the majority value $m$, where $m \in\{0,1\}$. Condition (3): if the majority does not exist then output the default value $\phi$.

\subsubsection{Calculating the Consensus Value for Return Vehicles}

When a return vehicle returns to the VANET, if it is a correct vehicle, we have to ensure that the consensus value it obtains conforms to the value obtained by other correct vehicles that have never been away. Our method is that the return vehicle will request the consensus values from vehicles that have never been away. Once it has collected $\left\lfloor\left(n_{O^{-}} a_{O^{-}}-1\right) / 3\right\rfloor+1$ uniform values, it will set this uniform value, denoted by $\mathcal{V}$, as its consensus value.

\section{Conclusion \& Future Work}

The development of ITS can provide us with a safer, more efficient and convenient life. Hence, fault tolerance of VANETs is of high importance, and it is necessary to investigate the consensus problem in VANETs. In this paper, we focus on the consensus problem in V2V-based VANETs. Through the proposed consensus protocol, all the correct vehicles can reach a consensus value in V2V-based VANETs without influence from Byzantine faulty vehicles. In our future work, we will concentrate on the consensus problem in V2R-based VANETs. Unlike V2V communications, V2R communications rely on the infrastructure (i.e. RSUs). RSUs can access the back-end network and provide a powerful computing capability. Therefore, we will address how to use RSUs to help OBUs obtain an agreement in our future work.

\section{References}

[1] J. Alcaraz, J. Vales-Alonso, and J. Garcia-Haro. Control-based scheduling with QoS support for vehicle to infrastructure communications. IEEE Wireless Commun. 2009, 16 (6): 32-39.

[2] M. Barborak, M. Malek, and A. Dahubra. The consensus problem in fault-tolerant computing. ACM Comput. Surv. 1993, 25 (2): 171-220.

[3] B.K. Chaurasia, and S. Verma. Secure pay while on move toll collection using VANET. Comput. Stand. Inter. 2014, 36 (2): 403-411.

[4] T.W. Chim, S.M. Yiu, C.K. Hui, and O.K. Li. VSPN: VANET-based secure and privacy-preserving navigation. IEEE Trans. Comput. 2014, 63 (2): 510-524.

[5] J.M. Chung, M. Kim, Y.S. Park, M. Choi, S.W. Lee, and H.S. Oh. Time coordinated V2I communications and handover for WAVE networks. IEEE J. Sel. Areas Commun. 2011, 29 (3): 545-558.

[6] M. Correia, A.N. Bessani, and P. Veríssimo. On Byzantine generals with alternative plans. J. Parallel Distr. Com. 2008, 68: 1291-1296.

[7] M.D. Dikaiakos, A. Florides, T. Nadeem, and L. Iftode. Location-aware services over vehicular ad-hoc networks using car-to-car communication. IEEE J. Sel. Areas Commun. 2007, 25 (8): 1590-1602.

[8] M. Fischer, and N. Lynch. A lower bound for the time to assure interactive consistency. Inform. Process. Lett. 1982, 14 (4): 183-186.

[9] M. Fischer, M. Paterson, and N. Lynch. Impossibility of distributed consensus with one faulty process. J. ACM. 1985, 32 (4): 374-382.

[10] M. Gramaglia, M. Calderon, and C.J. Bernardos. ABEONA monitored traffic: VANET-assisted cooperative traffic congestion forecasting. IEEE Veh. Technol. Mag. 2014, 9 (2): 50-57.

[11] S. Jafar, A. Krings, and T. Gautier. Flexible Rollback Recovery in Dynamic Heterogeneous Grid Computing. IEEE Trans. Depen. Secure. 2009, 6 (1): 32-44.

[12] Y. Jeong, J.W. Chong, H. Shin, and M.Z. Win. Intervehicle communication: cox-fox modeling. IEEE J. Sel. Areas Commun. 2013, 31 (9): 418-433.

[13] L. Lamport, R. Shostak, and M. Pease. The Byzantine generals problem. ACM Trans. Progr. Lang. Sys. 1982, 4 (3): 382-401.

[14] W.H. Lee, S.S. Tseng, and C.H. Wang. Design and implementation of electronic toll collection system based on vehicle positioning system techniques. Comput. Commun. 2008, 31 (12): 2925-2933. 
[15] T. Ma, J. Hillston, and S. Anderson. On the quality of service of crash-recovery failure detectors. IEEE Trans. Depen. Secure. 2010, 7 (3): 271-283.

[16] M. Oche, R.M. Noor, and J.I. Aghinya. Network centric QoS performance evaluation of IPTV transmission quality over VANETs. Comput. Commun. 2015, 61: 34-47.

[17] M. Pease, R. Shostak, and L. Lamport. Reaching agreement in the presence of faults. J. ACM. 1980, 27 (2): $228-$ 234.

[18] C. Rezende, A. Boukerche, H.S. Ramos, and A.A.F. Loureiro. A reactive and scalable unicast solution for video streaming over VANETs. IEEE Trans. Comput. 2015, 64 (3): 614-626.

[19] A. Wasef, R. Lu, X. Lin, and X. Shen. Complementing public key infrastructure to secure vehicular ad hoc networks. IEEE Wireless Commun. 2010, 17 (5): 22-28.

[20] W. Wu, J. Zhang, A. Luo, and J. Cao. Distributed mutual exclusion algorithms for intersection traffic control. IEEE Trans. Parallel Distrib. Syst. 2015, 26 (1): 65-74.

[21] M.B. Younes, A. Boukerche, and G. Rom'an-Alonso. An intelligent path recommendation protocol (ICOD) for VANETs. Comput. Netw., 2014, 64: 225-242.

[22] J. Zhou, Q. Hu, and Y. Qian. A scalable vehicular network architecture for traffic information sharing. IEEE J. Sel. Areas Commun. 2013, 31 (9): 85-93. 